\title{
Ultrafast Infrared Nanoscopy with Sub-Cycle Temporal Resolution
}

\author{
Tyler L. Cocker ${ }^{1}$, Max Eisele ${ }^{1}$, Markus A. Huber ${ }^{1}$, Markus Plankl ${ }^{1}$, Leonardo Viti ${ }^{2}$, Daniele Ercolani ${ }^{2}$, \\ Lucia Sorba ${ }^{2}$, Miriam S. Vitiello² and Rupert Huber ${ }^{1}$ \\ 1. Department of Physics, University of Regensburg, 93040 Regensburg, Germany \\ 2. NEST, CNR - Istituto Nanoscienze and Scuola Normale Superiore, 56127 Pisa, Italy
}

Ultrafast microscopy of surfaces with simultaneous nanometer spatial resolution and femtosecond temporal resolution can be achieved by coupling ultrafast optical pulses to sharp metal tips [1-10]. In the case of scattering-type near-field scanning optical microscopy (s-NSOM), evanescent fields scattered from the tip apex provide a window into a drastically subwavelength world. Moreover, since these scattered fields can be measured using established far-field technologies, ultrafast infrared pulses emerging from a nanoscale volume can be detected with the best possible time resolution: faster than a single optical oscillation cycle [1].

Sub-cycle time resolution in the far- and mid-infrared can be achieved by ultrabroadband field-resolved terahertz spectroscopy [11,12]. This spectral range contains a host of important collective excitations in condensed matter systems, including plasmons, phonons and magnons. Both the dynamics and character of these low-energy excitations can change significantly as the dimensions of a system are reduced to the nanoscale. Their properties can also vary between different nanoparticles in an ensemble, as nanoparticle size, structure, and orientation is often heterogeneous. Direct, ultrafast measurements within single nanoparticles are thus of great interest.

Here, we demonstrate a unique combination of ultrafast multi-terahertz (mid-infrared) spectroscopy with s-NSOM. Phase-stable multi-terahertz pulses are coupled to the tip of an s-NSOM and the scattered radiation is detected by electro-optic sampling. We record the oscillating electric near field with a time resolution given by the duration of the electro-optic gate pulse (10 fs, sub-cycle). Meanwhile, the radius of curvature of the s-NSOM tip apex defines our nano-spectroscopy spatial resolution (10 nm).

We have applied our novel microscope to the study of ultrafast local carrier dynamics in a single, isolated indium arsenide nanowire. Ultrafast imaging of the scattered near-field intensity (Fig. 1(a) and 1(b)) provides a map of the photoinduced carrier density, while field-resolved nano-spectroscopy (Fig. 1(c)) reveals the local evolution of the plasma frequency with sub-cycle time resolution. The plasma frequency manifests itself as a resonance in our probe spectrum, and can be used to calculate the instantaneous local carrier density at the surface of the nanowire. The carrier population dynamics feature a decay on the picosecond scale, which agrees well with typical carrier trapping times. Surprisingly, though, we also observe a very fast initial decay ( $<50 \mathrm{fs}$ ). To reveal its origin we have employed a novel technique we call femtosecond tomography. This approach allows us to trace the electron dynamics at different probing depths inside the material. The ultrafast decay is found to only occur close to the surface of the wire and can be explained by the ultrafast build-up of a surface depletion layer in the nanowire [13]. 
References:

[1] M Eisele et al, Nature Photon. 8 (2014) 841.

[2] S Berweger et al, Nano Lett. 11 (2011) 4309.

[3] J M Atkin et al, Adv. Phys. 61 (2012) 745.

[4] M Wagner et al, Nano Lett. 14 (2014) 894.

[5] M Wagner et al, Nano Lett. 14 (2014) 4529.

[6] Y Terada et al, Nature Photon. 4 (2010) 869.

[7] S W Wu and W Ho, Phys. Rev. B 82 (2010) 085444.

[8] A Dolocan et al, J. Phys. Chem. C 115 (2011) 10033.

[9] T L Cocker et al, Nature Photon. 7 (2013) 620.

[10] S Yoshida et al, Nature Nanotech. 9 (2014) 588.

[11] R Ulbricht et al, Rev. Mod. Phys. 83 (2011) 543.

[12] P U Jepsen et al, Laser Photon. Rev. 5 (2011) 124.

[13] The authors thank M. Furthmeier for technical assistance. This work was supported by the European Research Council through ERC grant 305003 (QUANTUMsubCYCLE), the Deutsche Forschungsgemeinschaft through Graduate Research College GRK 1570, and the Italian Ministry of Education, University, and Research (MIUR) through the Futuro in Ricerca 2010 grant RBFR10LULP (Fundamental Research on Terahertz Photonic Devices). T.L.C. acknowledges the support of the Alexander von Humboldt Foundation.

(a)
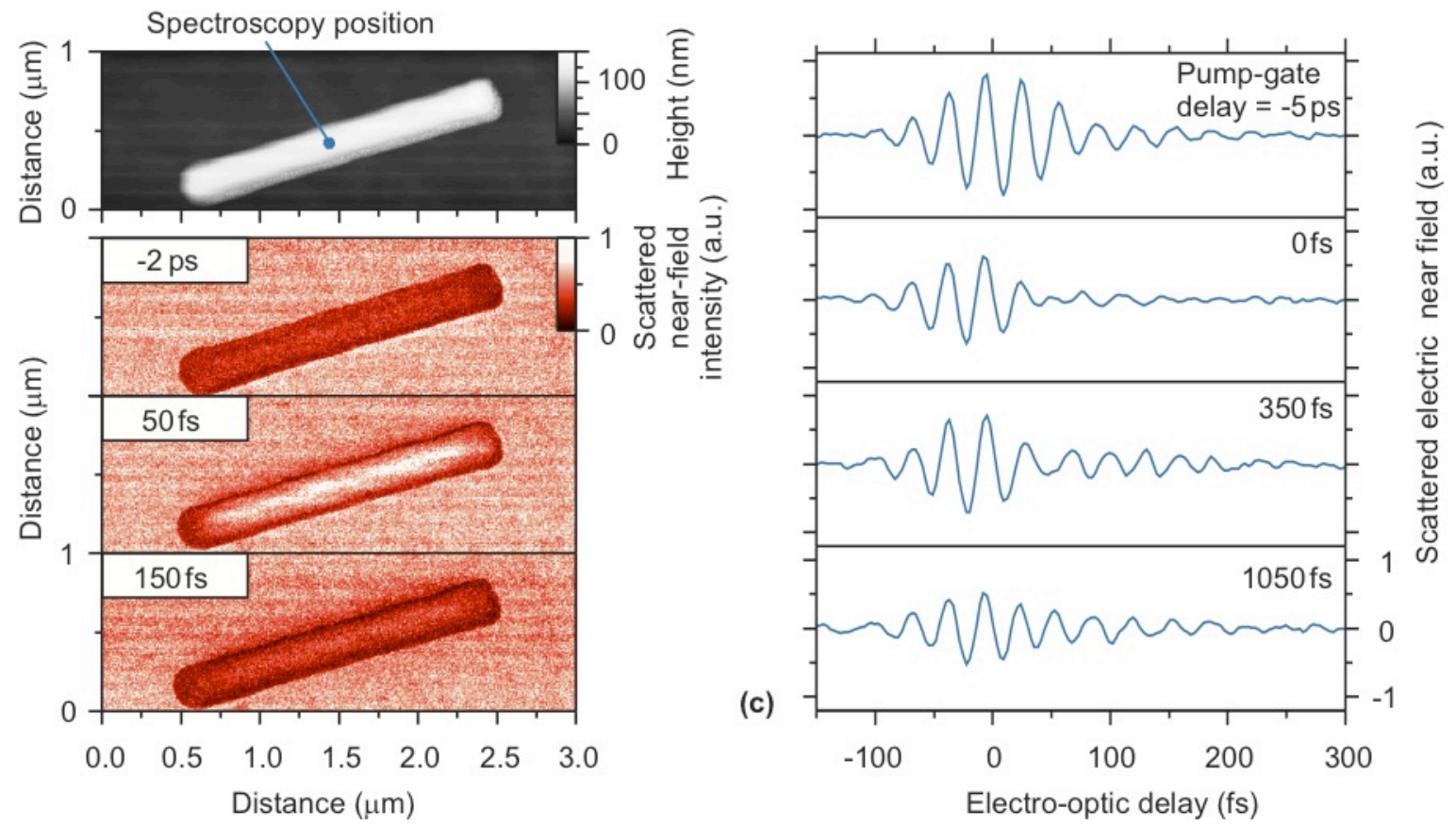

Figure 1. Pump-probe multi-THz spectroscopy and microscopy of a single InAs nanowire. (a) Topography of the InAs nanowire recorded by AFM. (b) Scattered intensity of the multi-THz probe pulse as a function of near-IR pump/multi-THz probe delay time and AFM tip position. (c) Scattered near-field waveforms recorded at the spectroscopy position denoted in (a). The oscillating electric near field from a $(10 \mathrm{~nm})^{3}$ volume is traced with $10 \mathrm{fs}$ temporal resolution 